\title{
Self-Organized Complementary Coordination: Dynamics of an Interpersonal Collision-Avoidance Task
}

\author{
Michael J. Richardson *, Steven J. Harrison", Ryan May*, \\ Rachel W. Kallen ${ }^{*}$ and R. C. Schmidt ${ }^{{ }^{*} \dagger}$. \\ *Center for Cognition, Action, \& Perception and University of Cincinnati, USA \\ ${ }^{*}$ Department of Psychology, College of the Holy Cross, USA \\ Email: richamo@ucmail.uc.edu,harri2sv@ucmail.uc.edu,mayrn@mail.uc.edu, \\ kallenrl@ucmail.uc.edu, rschmidt@holycross.edu
}

\begin{abstract}
The current study investigated the movement dynamics of a complementary collision-avoidance task. Participant pairs performed a repetitive targeting task in which they moved computer stimuli back and forth between sets of target locations without colliding into each other. Pairs converged onto a stable relative phase relationship of around $35^{\circ}$, with one participant spontaneously adopting the role of 'phase leader' and the other participant adopting the role of 'phase follower'. In addition, those participants who adopted the role of phase leader tended to exhibit more of a straight-line trajectory between targets, whereas participants who adopted the role of phase follower tended to exhibit a more elliptical trajectory. This role differentiation was essential to task success and reflected the spontaneous relaxation and recruitment of available movement degrees-of-freedom defined across the joint-action system.
\end{abstract}

\section{Introduction}

Many of our actions are performed together with other individuals and can be characterized as interpersonal or joint actions. Although there is a growing body of research investigating the neural and cognitive mechanisms that play a role in joint action, identifying the dynamical processes by which individuals are mutually responsive to one another in time and space is also crucial to understanding such behavior. Although past research has investigated the dynamics of the joint coordination for both intended and spontaneous between-person interactions, it has only investigated the presence of these processes in simple rhythmic coordination tasks, which included movements that are coordinated incidentally in 1-to-1 manner [see 1 for a review]. Accordingly, the current study investigated the dynamics of a more complex joint action in which individuals had to perform goaldirected movements without colliding into each other. We expected that complementary temporal and spatial patterns of the movement trajectories would reveal how co-actors play different dynamical roles in the performance of such joint-action tasks.

\section{Method}

Twelve pairs participated in the experiment. Participant pairs were instructed to perform a repetitive targeting task in which they each moved a computer stimulus back and forth between sets of target locations without the stimuli colliding into each other. Each participant in a pair stood facing a 50 " computer monitor with the screens positioned so that participants could not see each other (Fig. 1).

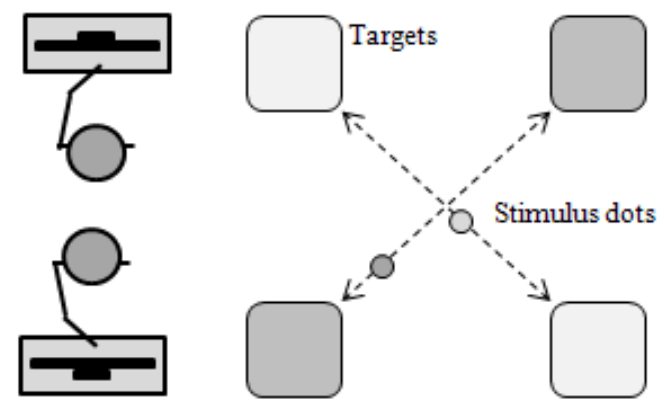

Fig.1. Collision-Avoidance setup.

The stimulus controlled by each participant was a small red dot $5 \mathrm{~cm}$ in diameter. The targets were $\sim 20 \mathrm{~cm}$ square and were positioned in each of the four corners of the monitor, with one participant moving their stimulus between the bottom-left and top-right target set and the other participant moving their stimulus between the bottom-right and top-left target set (Fig. 1). A magnetic motion tracking system (Polhemus Ltd) was used to record and track the movements of each participant. Participants held

This is an Open Access article distributed under the terms of the Creative Commons Attribution-Noncommercial License 3.0, which permits unrestricted use, distribution, and reproduction in any noncommercial medium, provided the original work is properly cited. 
a motion tracking sensor in their dominant hand to control the motion of the computer-generated stimulus in real-time. Each monitor displayed the real-time motion of the participant's own stimulus, as well as the motion of their co-participant's stimulus.

Upon arrival participants were informed that the experiment was investigating joint action, that each of them would be required to control and move a computer stimulus and that "the goal of the task is to move your stimulus back and forth between your target locations continuously and at a comfortable speed and to coordinate your movements with each other so that your stimuli do not collide, hit, or bump into each other". No other instructions as to the pattern or form of the coordination were provided. Participants were informed that the trial-by-trial procedure was designed as a game and that if they continuously moved between their respective targets without their stimuli colliding into each other for the entire length of a trial-40 seconds-they would receive 1 point. The goal was to achieve a score of 15 (i.e., complete 15 successful trials).

\section{Results}

One pair was unable to complete the task within the experimental session and was therefore dropped from the analysis. On average, the pairs took 21 trials to reach a score of 15 (range 15-36), with 8 of the 11 pairs reaching a score of 15 in less than 22 trials. Given the relatively small number of failed trials and the fact that $69 \%$ of failures occurred within the first 20 s of a trial (mean failure time of $14.65 \mathrm{~s}$ ), only data from successful trials is reported here.

Prior to analysis, the movement time-series from successful trials were low-pass filtered using a $10 \mathrm{~Hz}$ Butterworth filter. The movement and coordination patterns that emerged during successful task completion were determined using 1) principle components analysis, 2) measures of relative phase and 3) coupling directionality.

\subsection{Principle Components Analysis (PCA)}

PCA was used to calculate the normalized spread or width $(\delta)$ of each participant's movement about the principle axis of movement. In short, $\delta$ equals the ratio of the eigenvalues $(\lambda)$ from the covariance matrix between a participants $\mathrm{x}$ (frontal) and $\mathrm{y}$ (sagittal) data (i.e., $\delta=\lambda_{2} / \lambda_{1}$ ). As a ratio of the excursions perpendicular to the principle axis of motion, $\delta$ provides a measure of movement 'straightness' or 'spread' relative to the angular direction of motion [2]. With respect to the current data, the larger the value of $\delta$ the greater the deviation from a straight line movement trajectory (the more circular a participant's movement).

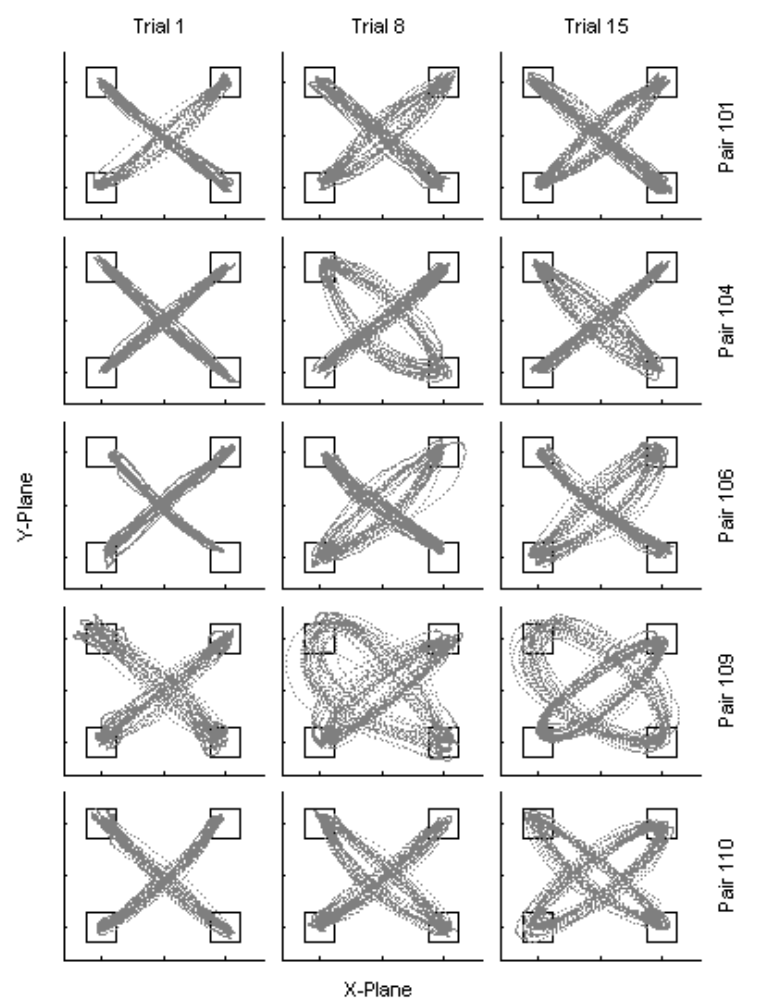

Fig.2. Example movement data. Trials 1, 8 and 15 for five different pairs.

The importance of $\delta$ for understanding the movement patterns adopted by participants to successfully perform and complete the task can be seen from an inspection of Fig. 2, in which the movement time-series trajectories for trials 1,8 and 15 for five different pairs are displayed. The data is a representative sample of the movement patterns observed across all pairs and captures the characteristic changes in the trajectories of participants from the first to the last successful trial. Two distinct features should be noted from Fig. 2 . First, one or both participants in a pair tended to adopt a more circular or oval movement trajectory. Indeed, a one-way repeated measures ANOVA comparing mean $\delta$ for the first, middle and last trials blocks was significant, $F(2,20)=8.40, p<.01, \eta_{p}{ }^{2}=$ .47 , with Bonferroni post-hoc test revealing that the first trial block was significantly lower than the middle and last trial blocks ( $p<.05$; see Fig. 3$)$.

Second, one participant in a pair adopted a greater degree of movement circularity than their coparticipant. That is, across trials one participant in a pair adopted a straight or straighter movement trajectory between targets, whereas the other participant adopted a more circular movement 
trajectory between targets. For 9 of the 11 pairs, this movement trajectory differentiation remained constant or increased across trials. A close inspection of the data revealed that this inter-participant movement differentiation occurred within the first 1 to 4 trials and remained that way for the remainder of the experimental session. Interestingly, for the two pairs in which the two participants exhibited approximately an equal number of trials with the greater magnitude of curvature, it was still the case that on any given trial one participant adopted a more curvilinear motion between targets.
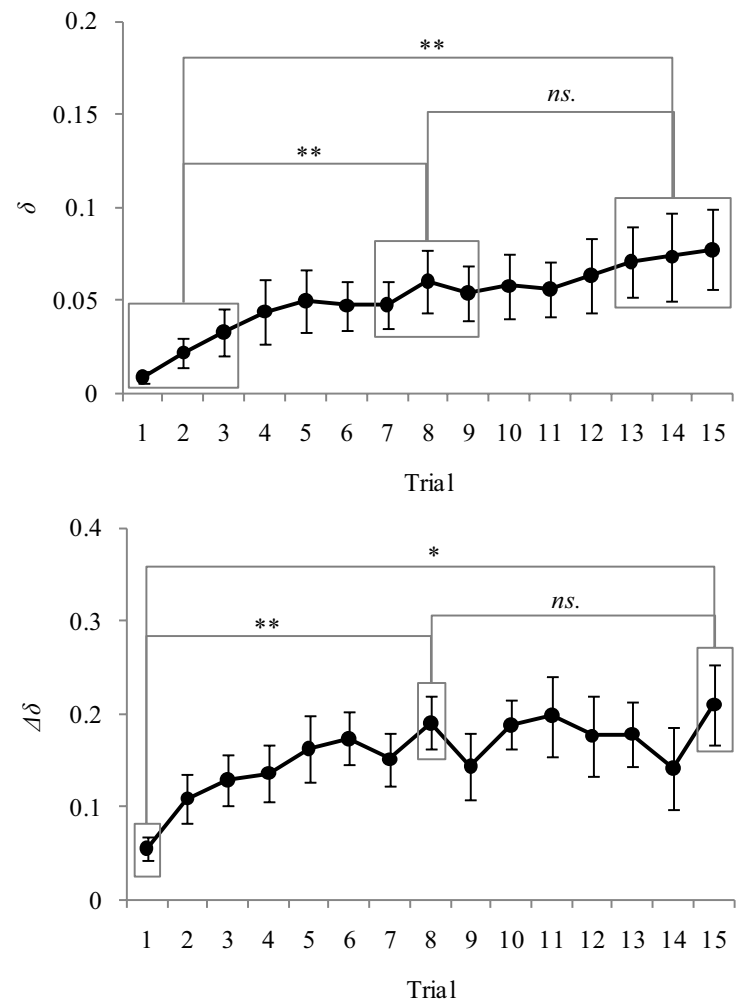

Fig.3. Mean $\delta$ and $\Delta \delta$ as a function of trial. Error bars indicate standard errors from the mean $(* p$ $<.05 ; * *$ p $<.01 ;$ ns. $p>.05)$.

To further determine whether the increased interparticipant differentiation in movement curvature was a significant factor across trials, a normalized $\delta$ difference score was calculated as $\Delta \delta=\sqrt{\left|\delta_{A}-\delta_{A}\right|}$. The resulting $\Delta \delta$ scores are displayed in Fig. 3 and although there was an increase in the between pair variability of $\Delta \delta$ across trials, there was also a clear increase in $\Delta \delta$. The increased variability across trials, however, led to only a marginally significant, $F(2$, $20)=3.02, p=.07, \eta_{p}{ }^{2}=.23$ difference between the average $\Delta \delta$ for the first, middle and last trial blocks. Given that pairs appeared to learn to differentiate their movement trajectories within the first few trials, a subsequent one-way ANOVA comparing $\Delta \delta$ for trials 1,8 and 15 was also performed. This latter analysis was found to be significant, $F(2,18)=$ 11.37, $p<.01, \eta_{p}{ }^{2}=.53$, with Bonferroni post-hoc tests revealing that $\Delta \delta$ was significantly lower for trial 1 compared to trials 8 and 15 (both $p<.02$ ).

\subsection{Relative Phase Analysis}

Given the PCA results, the relative phase, $\phi$, of a pairs movement was calculated (using the Hilbert transform) with respect to the participants' principle axis of their $\mathrm{x} / \mathrm{y}$ movements (i.e., along the diagonal). The phase of the participant with the greater value of $\delta$ for the corresponding trial was subtracted from the phase of the participant with the lower value of $\delta$ (i.e., $\phi=\theta_{\delta<}-\theta_{\delta>}$ ).

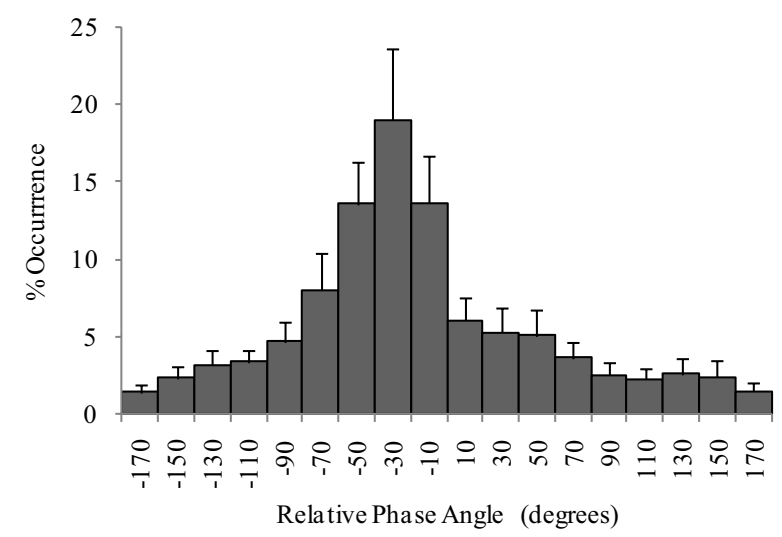

Fig.4. Distribution of relative phase (DRP).

Due to the fact that participants started each trial at their bottom targets, $\phi=0^{\circ}$ corresponded to participants moving to their top and bottom targets at the same time, whereas $\phi=180^{\circ}$ corresponded to participants moving to vertically opposite targets at the same time. It is important to note that the latter statements of what corresponded to 'inphase' and 'antiphase' movement is for the purposes of clarity only. Neither of these canonical relative phase modes were expected to be observed during successful task performance, as prototypical $0^{\circ}$ or $180^{\circ}$ relative phase relations would have resulted in a collision. Given the latter expectation and the fact that the stable relative phase relations that occurred were more variable than typically observed in rhythmic coordination studies, the distribution of the relative phase (DRP) was employed as a measure of the stability and patterning of relative phase. DRP was determined by calculating the percentage of occurrence of relative phase angles across eighteen $20^{\circ}$ regions of relative phase between $0^{\circ}$ and $360^{\circ}$. 
Negative angles correspond to the participant with the greater $\delta$ (the more circular trajectory) lagging behind the phase of the participant with the lower $\delta$ (the straighter circular trajectory).

As expected, participants did not coordinate in a canonical inphase pattern (see Fig. 4). Rather participants who adopted a more circular movement trajectory typically phase lagged or followed the participants with the straighter or less circular movement trajectory by approximately $35^{\circ}$ to $45^{\circ}$.

\subsection{Directionality of Coupling}

Finally, we estimated the directionality of the coupling between the participants using the evolution map approach proposed by [3]. The method provides a measure of the directionality of the coupling between two self-sustained oscillatory movements by estimating the ratio of the coupling terms from the phase time series of the movements. This directionality index, $d_{(1,2)}$, varies from 1 in the case of unidirectional coupling $\left(\theta_{1} \rightarrow \theta_{2}\right)$ to -1 in the opposite case $\left(\theta_{2} \rightarrow \theta_{1}\right)$, with $d_{(1,2)}=0$ corresponding to symmetric bidirectional coupling $\left(\theta_{2} \leftrightarrow \theta_{1}\right)$. Note that $d_{(1,2)}$ estimates of how strongly each oscillator is driven by the other. Thus, $d_{(1,2)}=1$ indicates a unidirectional coupling, whereby $\theta_{2}$ is driven by $\theta_{1}$.

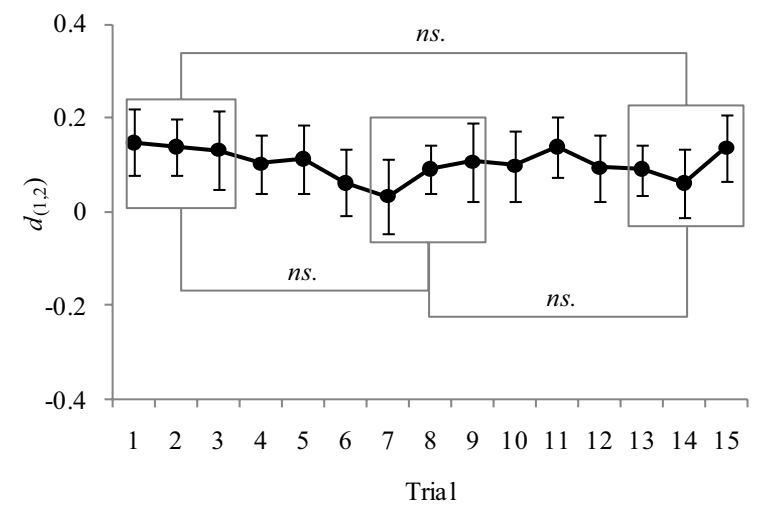

Fig.5. Index of coupling directionality, $d_{(1,2)}$.

As with the calculations of relative phase above, the directionality index, $d_{(1,2)}$, was calculated for each trial with respect to the values of $\delta$, with the phase time series of the participant with the greater value of $\delta$ corresponding to $\theta_{1}$ and the phase time series of the participant with the lower value of $\delta$ corresponding to $\theta_{2}$. The average $d_{(1,2)}$ values as a function of trial are displayed in Fig. 5. Interestingly, there was a small but consistent asymmetry in the coupling between $\theta_{1}$ and $\theta_{2}$, with slightly positive $d_{(1,2)}$ values for each trial. There was no difference in $d_{(1,2)}$ between the first, middle and last trial blocks, $F(2,20)<1$, and $d_{(1,2)}$ averaged across all trials was significantly different from zero, $t(10)=3.46, p<.01$. This indicates that on any given trial, the participant with the more circular trajectory was coupled (driven) more strongly to the movements of the participant with the straighter trajectory. In other words, the phase-leader to phase-follower coupling was weaker than phase-follower to phase-leader coupling.

\section{Conclusion}

The patterns of movement coordination observed in this collision-avoidance experiment provide initial evidence that the sub-roles that emerge during joint action are a consequence of individuals differentially recruiting available degrees-of-freedom to stabilize task performance. Indeed, learning to appropriately relax, recruit, and suppress different movement degrees-of-freedom is essential to task-specific flexibility and often leads to a more stable and perturbation-resistant state of coordination [e.g., 4, 5]. Moreover, it is this kind of action differentiation that defines a task as complementary, in that the different movements of the individuals sustain the most stable and effective way to 'complete' a mutual task. Therefore, task success is not reducible to the movements of any one individual [e.g., 6, 7], but rather is dependent on the collective organization of the joint actions system as a whole.

\section{References}

[1] Schmidt, R. C., \& Richardson, M. J. (2008). Dynamics of interpersonal coordination. In A. Fuchs \& V. Jirsa (Eds.) Coordination: Neural, Behavioral and Social Dynamics (pp. 281-308). Germany: Springer.

[2] Duarte, M., \& Zatsiorsky, V. M., (2002). Effects of body lean and visual information on the equilibrium maintenance during stance. Experimental Brain Research, 146, 60-69.

[3] Rosenblum, M. G., \& Pikovsky, A. S. (2001). Detecting direction of coupling in interacting oscillators. Physical Review E, 64, 45202-1-45202-4.

[4] Fink, P., Kelso, J. A. S., \& DeGuzman, G.C. (2000). Recruitment of degrees of freedom stabilizes coordination. Journal of Experimental Psychology: Human Perception and Performance, 26, 671-692.

[5] Richardson, M. J., Campbell, W. L. \& Schmidt, R. C. (2009). Movement interference during action observation as emergent coordination. Neuroscience Letters, 449, 117-122.

[6] Riley, M. A., Richardson, M. J., Shockley, K. \& Ramenzoni, V. C. (2011). Interpersonal synergies. Frontiers in Psychology, 2, 1-7.

[7] Theiner, G., Allen, C., Goldtsone, R. L., (2010). Recognizing group cognition. Cognitive Systems Research, 11, 378-395. 